\title{
Tutorial on Beam Current Monitoring
}

\author{
Robert C. Webber \\ Fermi National Accelerator Laboratory ${ }^{*}$, P.O. Box 500, Batavia, Illinois 60510 USA
}

\begin{abstract}
This paper is a tutorial level review covering a wide range of aspects related to charged particle beam current measurement. The tutorial begins with a look at the characteristics of the beam as a signal source, the associated electromagnetic fields, the influence of the typical accelerator environment on those fields, and the usual means of modifying and controlling that environment to facilitate beam current measurement. Short descriptions of three quite different types of current monitors are presented and a quantitative review of the classical transformer circuit is given. Recognizing that environmental noise pick-up may present a large source of error in quantitative measurements, signal handling considerations are given considerable attention using real-life examples. An example of a successful transport line beam current monitor implementation is presented and the tutorial concludes with a few comments about signal processing and current monitor calibration issues.
\end{abstract}

\section{INTRODUCTION}

Beam current, bunch charge, and beam pulse shapes are fundamental parameters to be measured in any particle accelerator, storage ring or beam transport line. Devices to perform these measurements may or may not physically intercept the beam particles. In synchrotrons, storage rings, and many beam transport lines non-intercepting types of monitors are essential to minimize induced beam loss or emittance blow-up. These monitors sample the electromagnetic fields of the beam to produce a useful signal.

Beam monitors can be made to sense the electric field, the magnetic field, or some combination. Devices that rely primarily on interaction with the beam's magnetic field to measure beam current, charge, and pulse shape are generally termed beam current monitors. From an engineering viewpoint, the beam current monitor is usefully described in the formalism of transformer circuit theory. Beam current transformers and their application in the accelerator environment are the topics of this tutorial.

\section{THE BEAM AS A SIGNAL SOURCE}

A charged particle beam exhibits an electric current of magnitude

$$
i_{b}=e \cdot \lambda_{N} \cdot v
$$

where $e$ is the particle charge, $\hat{\lambda}_{N}$ the number of particles per unit length, and $v$ the particle velocity.

*Work supported by the U.S. Department of Energy under contract No. DE-AC02-76CH03000. 
The impedance of an electrical current source is defined as $\Delta \mathrm{V} / \Delta \mathrm{I}$, where $\Delta \mathrm{V}$ represents the change in source terminal voltage due to an external perturbation and $\Delta \mathrm{I}$ is the resulting change in the source output current. An ideal source exhibits infinite impedance, i.e. the output current is completely independent of perturbations at the source terminals. A relativistic beam is nearly such an ideal source. To produce a change in the beam current, the beam must be subjected to a longitudinal voltage that will initially change the particle velocity and therefore it's energy. The magnitude of that source impedance can be described by

$$
Z_{b}=\left(\frac{d i}{d V}\right)^{-1}=\left[\left(e \cdot \lambda_{N}\right) \cdot\left(\frac{d \beta c}{d E}\right) \cdot\left(\frac{d E}{d V}\right)\right]^{-1}=\left(e \cdot \lambda_{N} \cdot \beta \cdot c\right)^{-1} \cdot\left(\beta^{2} \cdot \gamma^{3} \cdot E_{o} / e\right)
$$

where $e$ is the electronic charge, $E_{o}$ the particle rest energy, $c$ the speed of light, and $\beta$ and $\gamma$ the normal relativistic parameters.

For a $500 \mathrm{~mA} 8 \mathrm{GeV}$ proton beam, this is $1.67 \mathrm{E} 12 \mathrm{ohms}$. High impedance indeed! Note that the impedance is nonlinear; it is inversely proportional to the current itself. This is understood with the realization that any particles seeing an applied voltage will slow down equally. The more particles, the larger the effective change in current.

\section{THE BEAM, ITS FIELDS, AND ENVIRONMENT}

The beam communicates with its environment through the electric and magnetic fields it carries. The assembly of charged particles forming the beam typically travels through an evacuated chamber bounded by electrically conducting metallic walls. The beam charge produces an electric field that in turn induces an image charge on the chamber wall. As the charged beam particles are in motion, they produce a magnetic field that induces the image charge on the wall to flow along. The resulting "wall currents" are, to first order, equal and opposite to the beam current.

Ampere's Law states that the magnetic field around a closed path, e.g. a circle around the beam tube, is proportional to the sum of all currents enclosed by the path. Therefore, to the extent that wall currents mirror the beam current, the magnetic field outside the beam tube is cancelled.

The field strength reduction corresponds to the attenuation of an electromagnetic wave propagating through a conductor. The characteristic length in which the wave amplitude is reduced by a factor of $e(-8.69 \mathrm{~dB})$ is termed the skin depth.

The skin depth in a non-magnetic, good conductor is

$$
\delta=\frac{\sqrt{10} \cdot 10^{3}}{2 \pi} \sqrt{\frac{\rho}{f}}
$$

where $\rho$ is the resistivity of the conductor and $f$ is the wave frequency.

Figure 1 is a skin depth chart for copper and stainless steel at selected frequencies. The attenuation through a material thickness, $t$, is $8.69 \cdot t / \delta \mathrm{dB}$. For example, at $10 \mathrm{Mhz}$, a typical $1 / 32$ " $(0.794 \mathrm{~mm})$ stainless beam tube wall $(6.1$ skin depths) will 
attenuate magnetic fields propagating to the exterior by $53 \mathrm{~dB}$. This is sufficient to clobber the sensitivity of a practical beam current monitor, but not so much as to render the beam signal invisible to a sensitive radio receiver! The shielding effectiveness of copper is $55 \mathrm{~dB}$ better than that of the same thickness of stainless steel.

\begin{tabular}{|l|l|l|l|l|l|}
\hline & $1 \mathrm{KHz}$ & $10 \mathrm{KHz}$ & $100 \mathrm{KHz}$ & $1 \mathrm{MHz}$ & $10 \mathrm{MHz}$ \\
\hline Copper & $2.1 \mathrm{~mm}$ & $0.66 \mathrm{~mm}$ & $0.21 \mathrm{~mm}$ & $0.066 \mathrm{~mm}$ & $0.021 \mathrm{~mm}$ \\
\hline 302 Stainless Steel & $13.3 \mathrm{~mm}$ & $4.2 \mathrm{~mm}$ & $1.3 \mathrm{~mm}$ & $0.42 \mathrm{~mm}$ & $0.13 \mathrm{~mm}$ \\
\hline
\end{tabular}

FIGURE 1. Skin Depth Chart.

Since the magnetic field of the beam is severely attenuated outside a continuous conducting vacuum chamber, a practical beam current monitor requires a "window to the beam". The monitor must be placed within the vacuum chamber walls or the conducting path in the chamber must be broken. To minimize mechanical complications associated with inserting a device into the vacuum, a non-conducting material, often ceramic, may be inserted electrically in series with an otherwise continuous beam tube section. This interruption of the conduction path along the beam tube forces wall currents to find a new path, potentially under the instrument designer's control, outside the vacuum chamber.
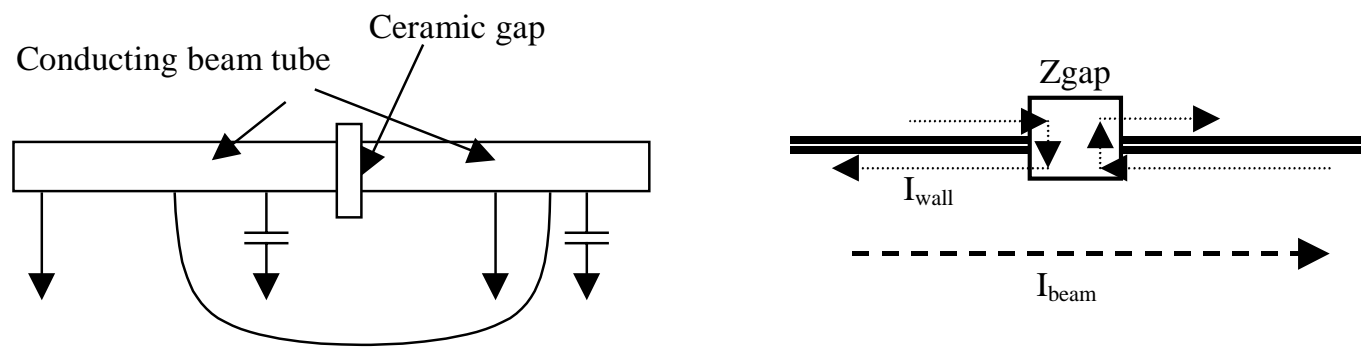

FIGURE 2. Typical ceramic gap installation in accelerator environment and equivalent circuit model.

Figure 2 depicts a ceramic break or ceramic gap installation in a typical beam tube. The break provides a local region suitable for placing a beam current monitor through which, at low frequencies at least, only the beam current flows. Wall currents take whatever paths, controlled or uncontrolled, that are presented across the gap. At low frequencies the lowest impedance path is often well out around the local region. The effective gap impedance is a parallel combination of the inherent gap capacitance and all external impedance, including beam tube capacitance, grounds, cable shields, straps or housings placed across the gap, and even the current monitor itself. Elements comprising the external paths may be intentional or incidental, local or distant, but will always be present. The beam will supply whatever gap voltage is required to drive the image currents through the effective gap impedance. A gap voltage

$$
V_{\text {gap }}(\omega)=Z_{\text {gap }}(\omega) \cdot I_{\text {wall }}(\omega)=Z_{\text {gap }}(\omega) \cdot I_{\text {beam }}(\omega)
$$

will be generated. 
Impedance measurements of a ceramic gap actually installed in the Fermilab $8 \mathrm{GeV}$ beam transport line are shown in Figure 3. The curve labeled "open" is the impedance of the gap as found. It shows that, in this case, a dc path with an inductance of about $1.1 \mathrm{uH}$ shunts the gap. A plot of the impedance of $1.1 \mathrm{uH}$ is overlaid for comparison. The measurement was not designed for quantitatively accurate results above a few tens of megahertz, but does serve to show that resonances can occur below the frequency where the gap capacitance begins to dominate. The "shorted" curve displays the result of placing shorting braids, long enough to reach outside and around a proposed beam current transformer, across the gap.

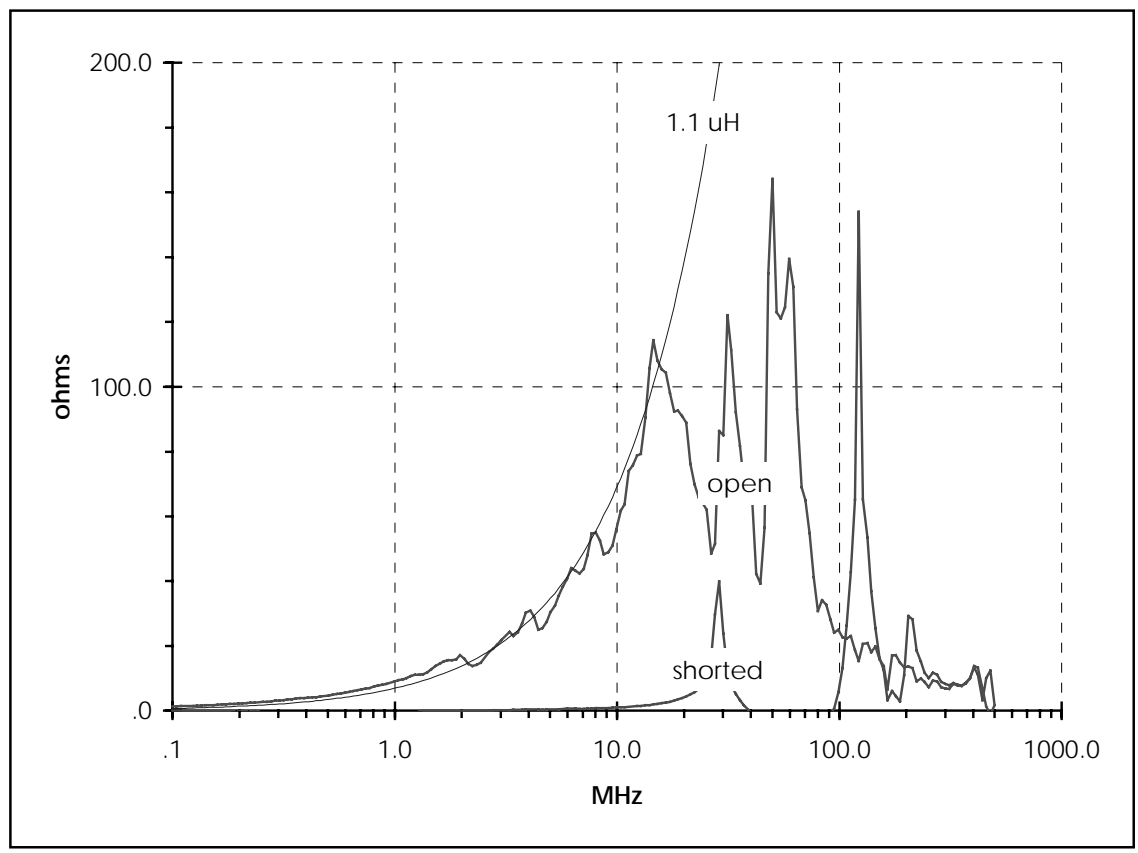

FIGURE 3. Impedance measurement of installed ceramic gap with and without a somewhat long shorting strap attached across the gap. Impedance of $1.1 \mathrm{uH}$ inductor included for reference.

Although a gap provides the required window to the beam, it may cause undesired side effects. The beam currents, typically with a broad frequency spectrum, will drive the gap impedance. Significant beam induced voltage can result if the gap environment is left uncontrolled. This voltage feeds back on the beam itself and can cause instabilities in circulating beams. The gap voltage and the currents taking paths external to the gap may couple into neighboring circuits and at high frequencies produce radiating fields. A beam current of $500 \mathrm{~mA}$ at $10 \mathrm{Mhz}$ through the impedance of the gap of Figure 3 will, for example, induce about 30 volts. This is insufficient to corrupt the beam in a small number of turns, but large enough to present itself as a 'noisy neighbor' to nearby circuits. Narrow, high impedance resonances can result in very high gap voltages and potentially serious beam disruption.

An example configuration to control side effects of gap impedance and improve the environment of a beam current monitor is shown in Figure 4. Straps or a full housing around the transformer and gap serve to reduce the effective impedance presented to 


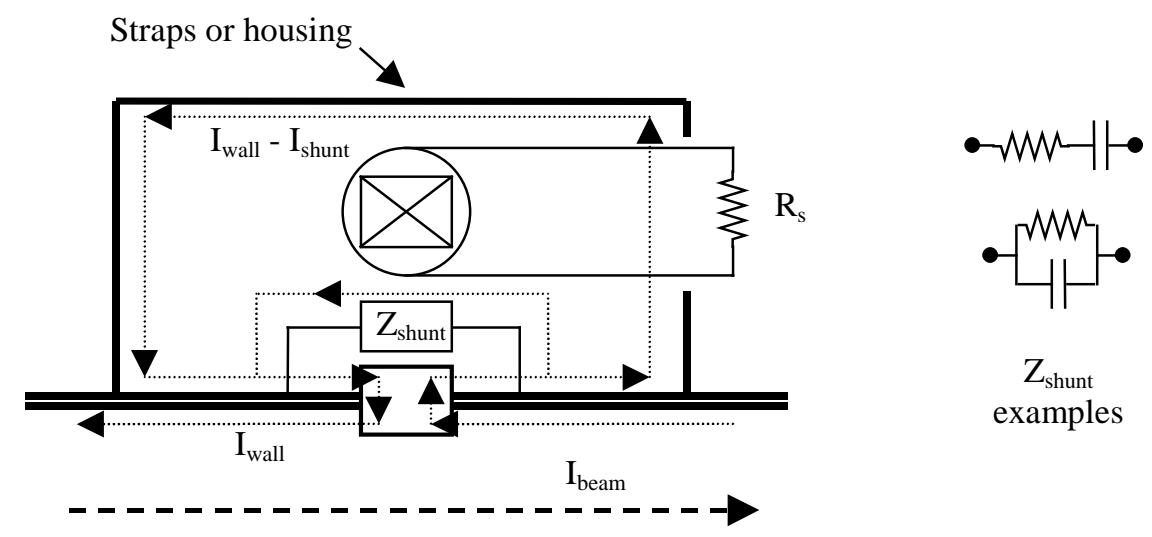

FIGURE 4. Model of current monitor enclosed in housing over gap with shunting elements to control impedance.

the beam (e.g. see the "shorted" curve in Fig. 3) and to shield the external world from the gap voltage and the wall currents. Resistor and capacitor circuits, $Z_{\text {shunt }}$, can be added to limit gap impedance and damp potential resonances. For best high frequency performance, multiple elements should be distributed across the gap in parallel and more or less uniformly around the circumference. The magnitude of $Z_{\text {shunt }}$ must be sufficiently high at frequencies where the beam current is to be measured so as not to short-circuit the gap as seen by the current monitor. Typically a value of $>10$ to 100 ohms is acceptable. For example, a 1 volt/amp transformer with an internal $50 \mathrm{ohm}$ termination presents a $0.02 \mathrm{ohm}$ impedance to the current being measured, therefore a $Z_{\text {shunt }}$ magnitude of 20 ohms over the measurement frequency range will cause only a $0.1 \%$ error. Either series or parallel resistor/capacitor circuits as shown in Figure 4 may be used. The series configuration provides a DC block to external noise or ground
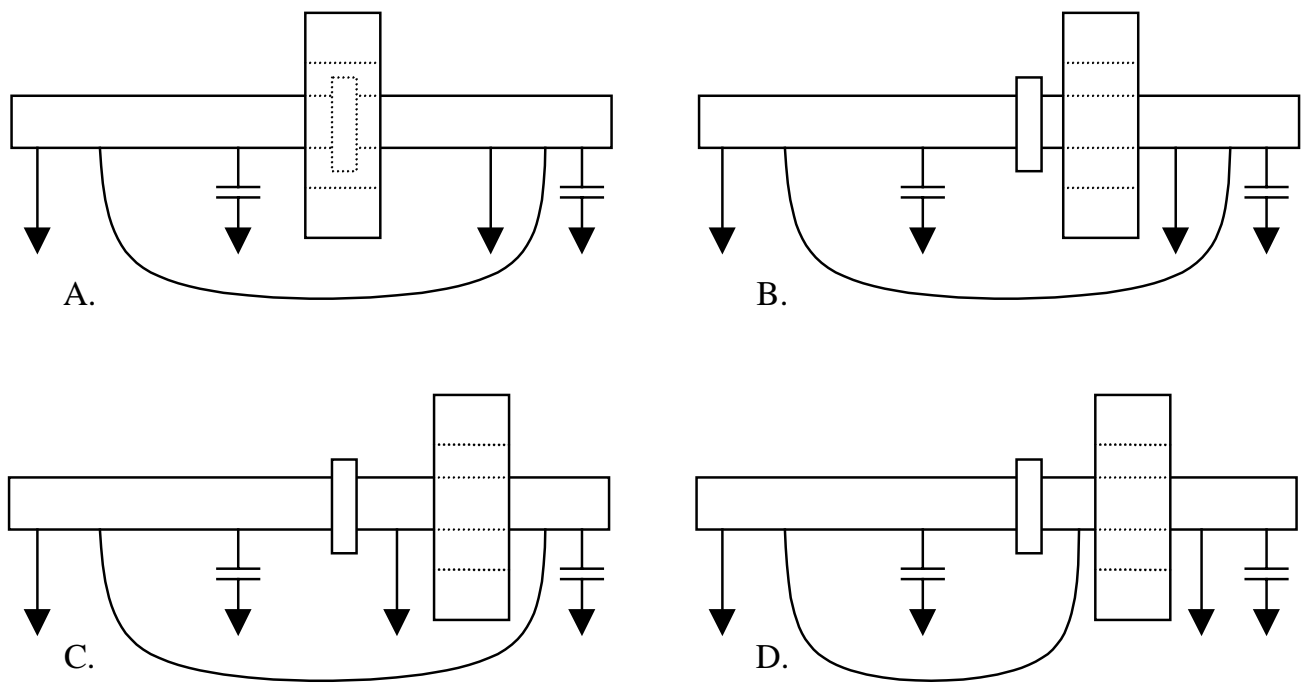

FIGURE 5. Potential locations of current monitor relative to beam tube gap. A and B depict acceptable locations, current bypass paths enclose the transformer. C and D show unacceptable locations because ground or other connections short-circuit the gap by paths not enclosing the monitor. 
currents that might attempt to flow through $\mathrm{Z}_{\text {shunt }}$ and appear as real signal to the monitor. If external strap or housing is present, these unwanted currents should not be a problem, as they will be short-circuited outside the monitor. The parallel configuration would appear to yield lower ultimate high frequency impedance, although at some point the inherent gap capacitance will dominate regardless.

A commonly asked question is "What is an acceptable position for a beam current monitor relative to the gap; does it need to go right over the gap?" The requirement is that all paths by which the wall current can bypass the gap must enclose the beam current monitor. The monitor should be placed as close to the gap as possible for best high frequency performance, but need not straddle the gap physically. Figure 5 shows four possible monitor locations. In each case, the wall current passes around the gap either via a ground path or some ungrounded path, perhaps two signal cable shields connected to the beam tube and also to another, possibly remote, common point. In Figures $5 \mathrm{~A}$ and $5 \mathrm{~B}$, those external paths enclose the beam current monitor and the wall current flows undetected outside the monitor. In Figures $5 \mathrm{C}$ and $5 \mathrm{D}$ the paths do not enclose the current monitor; the wall currents are observed by the current monitor and interfere with the beam current that is to be measured.

\section{COMMON TYPES OF BEAM CURRENT TRANSFORMERS}

Differing measurement requirements often require beam current monitors with special performance features. Three commonly used types of monitors, each with unique features and operating characteristics, are summarized.

\section{Integrating Current Transformer (ICT)}

The ICT is a passive current transformer. It operates on the principle that a sufficiently short $(<1 \mathrm{nsec})$ and isolated $( \pm 50 \mathrm{nsec})$ beam bunch driving the transformer's impulse response will produce an output pulse fixed in shape by the transformer design and independent of the beam pulse shape. The output pulse amplitude is directly proportional to bunch charge and is stretched in time compared to the bunch length.

The ICT is useful in synchrotrons, storage rings, and transport lines with short widely separated bunches. It is elegantly simple in design, easy to apply, relatively inexpensive, and offers stable passive calibration. The stretched output pulse eases timing requirements for signal sampling circuitry. A disadvantage of the ICT is that bunch shape information is not available; however, for the short bunches required for proper ICT operation a lumped element transformer is likely not the device of choice for bunch shape measurement in any case.

\section{Direct Current Transformer (DCCT, PCT, etc.)}

The DCCT and it siblings are active devices utilizing strong, precisely controlled AC magnetizing forces applied to one or more toroidal cores to enable the sampling of a magnetic bias imposed by beam or other current. This category of devices is unique in the ability to measure a DC current component. 
DCCTs operate in a zero average flux mode. Sensitive electronics detect any flux imbalance in the magnetic circuit and return a feedback current to precisely null that imbalance. When the initial flux is due to the magnetic bias induced by a beam current, the resulting feedback current will be directly proportional to that beam current. A stable, precision resistor in the feedback current path yields a voltage that serves as the output of the instrument. Practical DCCTs for particle beams are a combination DC section and AC transformer. This overcomes the aliasing inherent in any sampling circuit and extends the useful bandwidth of the system.

DCCTs are important in synchrotrons and storage rings where measurement of the DC $(0 \mathrm{~Hz})$ component of bunched or unbunched beams is important. These instruments can provide resolution and long-term stability at the level of one microampere DC. They are usually not appropriate for transport line applications and are relatively expensive for applications not requiring DC response.

\section{Classical AC Transformer}

The classical transformer functions as a beam current monitor with the beam acting as a single turn primary winding that drives magnetic flux in the transformer core to induce voltage in a secondary winding on same core. The output signal can provide a high fidelity representation of beam current pulses over a wide range of frequencies, pulse lengths, and amplitudes. The transformers are fundamentally passive devices, but may be supplemented with various active circuits to modify performance (e.g. Hereward and 'active-passive' configurations). Useful devices have been made for applications ranging from sub-hertz to multi-hundred megahertz frequencies.

Beam current monitors based on the classical transformer are simple and available in configurations to suit many applications. It is important to remember that the output is not DC coupled; no DC output component is present.

\section{Classical Transformer Review}

The classical transformer circuit is depicted in Figure 6. Current in any $N$ turn

winding $k$ produces magnetic flux in the core of magnitude $\Phi_{k}=\frac{L_{k} \cdot i_{k}}{N_{k}}$. The voltage appearing across each winding is proportional to the time rate change of the total flux, $\Phi_{T}$. Steady state equations of the Figure 6 circuit, in Laplace notation $(s=j \omega)$, are:

$$
\begin{gathered}
\Phi_{T}=\frac{L_{p} \cdot i_{p}}{N_{p}}-\frac{L_{s} \cdot i_{s}}{N_{s}} \\
i_{s}=\frac{s \cdot \Phi_{T} \cdot N_{s}}{R_{s}} \\
V_{g}=i_{p} \cdot R_{g}+s \cdot \Phi_{T} \cdot N_{p}
\end{gathered}
$$

Eq. 5 is the flux conservation equation. Eqs. 6 and 7 are the secondary and primary loop equations respectively. 


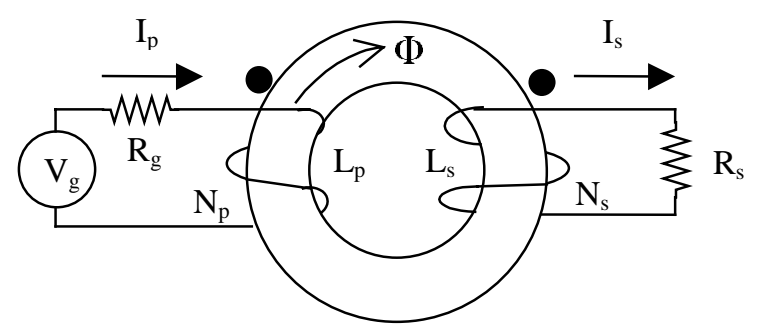

FIGURE 6. Classical transformer circuit.

Simultaneous solution of these equations yields

$$
\frac{V_{s}}{V_{g}}=\frac{i_{s} \cdot R_{s}}{V_{g}}=\frac{s \cdot L_{s} \cdot N_{p}}{R_{g} \cdot N_{s}} \cdot\left[1+s \cdot\left(\frac{L_{p}}{R_{g}}+\frac{L_{s}}{R_{s}}\right)\right]^{-1}
$$

In mid-band, where $s \cdot\left(\frac{L_{p}}{R_{g}}+\frac{L_{s}}{R_{s}}\right) \gg>1$, and using $\frac{L_{p}}{L_{s}}=\frac{N_{p}{ }^{2}}{N_{s}{ }^{2}}$, this simplifies to

$$
\frac{V_{s}}{V_{g}}=\frac{N_{s}}{N_{p}} \cdot\left(\frac{N_{p}^{2}}{N_{s}^{2}} \cdot R_{s}\right) \cdot\left(\frac{N_{p}^{2}}{N_{s}^{2}} \cdot R_{s}+R_{g}\right)^{-1}
$$

In the beam current model, it is appropriate to replace the voltage generator by an equivalent current generator,

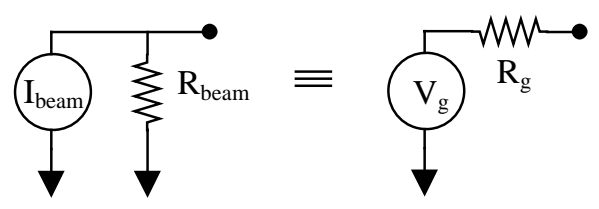

where $V_{g}=i_{\text {beam }} \cdot R_{\text {beam }}$ and $R_{g}=R_{\text {beam }}$. Substituting these relations into Eq. 9 with $N_{p}=1$, find

$$
\frac{i_{s}}{i_{\text {beam }}}=\frac{1}{N_{s}} \cdot\left(1+\frac{R_{s}}{N_{s}^{2} \cdot R_{\text {beam }}}\right)^{-1}
$$

For $R_{\text {beam }} \gg R_{s}$, the familiar approximate result, $i_{s}=\frac{i_{\text {beam }}}{N_{s}}$, is obtained. The secondary current is very closely equal to $1 / N_{s}$ times the beam current. 

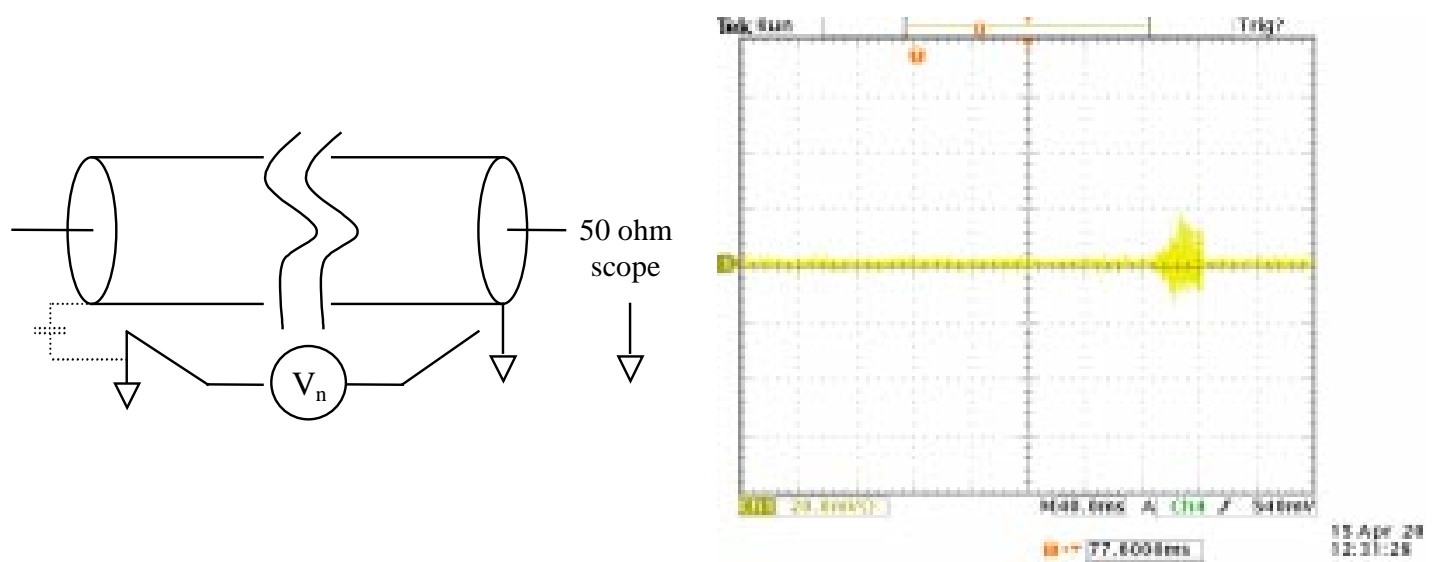

FIGURE 7. Noise measured on long ( 250 meter) RG8 coaxial cable into $50 \mathrm{ohms}$ with far end open and ungrounded. (20mV/div and $40 \mathrm{mS} / \mathrm{div})$

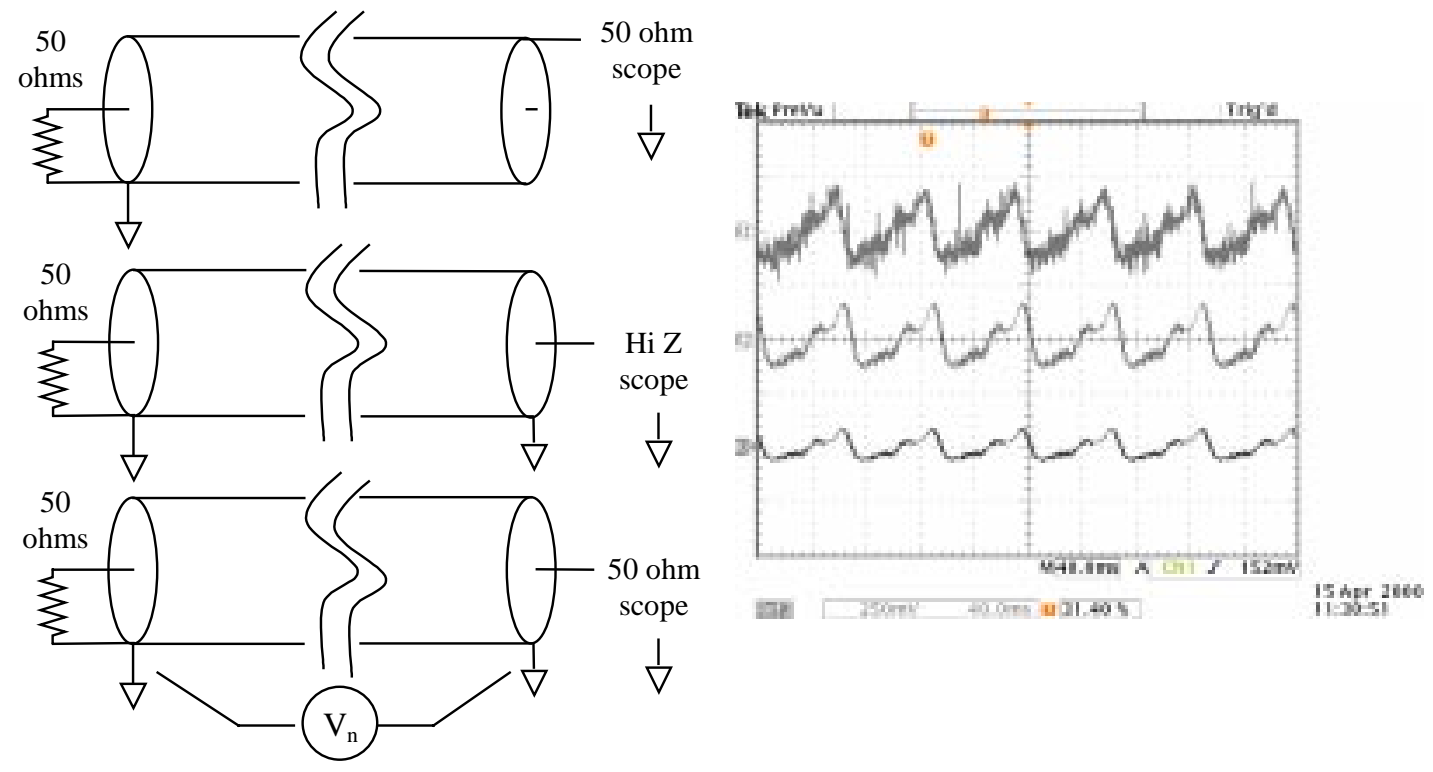

FIGURE 8. Noise on long RG8 cable with far end terminated and shield grounded. Top: noise on shield into $50 \mathrm{ohms}$. Middle: center conductor into high impedance. Bottom: center conductor into 50 ohms. (each $250 \mathrm{mV} / \mathrm{div}$ and $40 \mathrm{mS} / \mathrm{div}$ )

\section{NOISE ON CABLES}

Noise picked up on signal cables is a persistent problem in systems forced to operate in the accelerator environment and beam current monitors are no exception. Figures 7-11, 13, and 14 display noise observed on long coax and twinax cables at the 


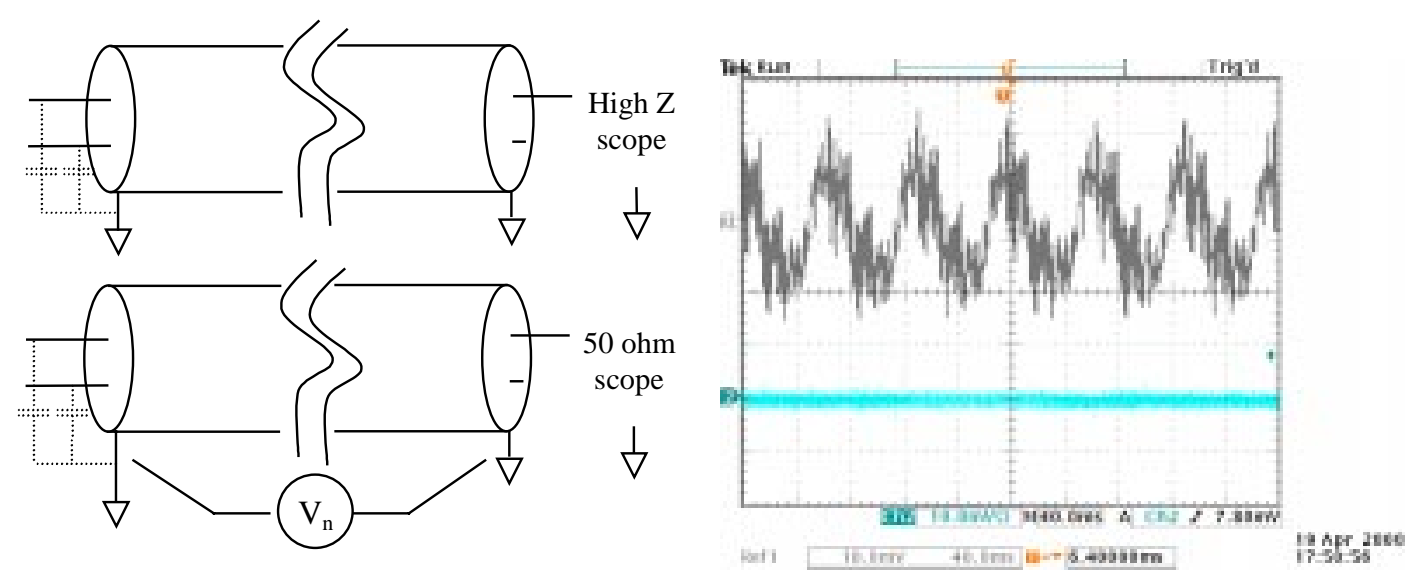

FIGURE 9. Noise on long ( 300 meter) twinaxial cable with far end center open and shield grounded. Top: noise on either center into high impedance. Bottom: either center into 50 ohms. (each $10 \mathrm{mV} / \mathrm{div}$ and $40 \mathrm{mS} / \mathrm{div}$ )

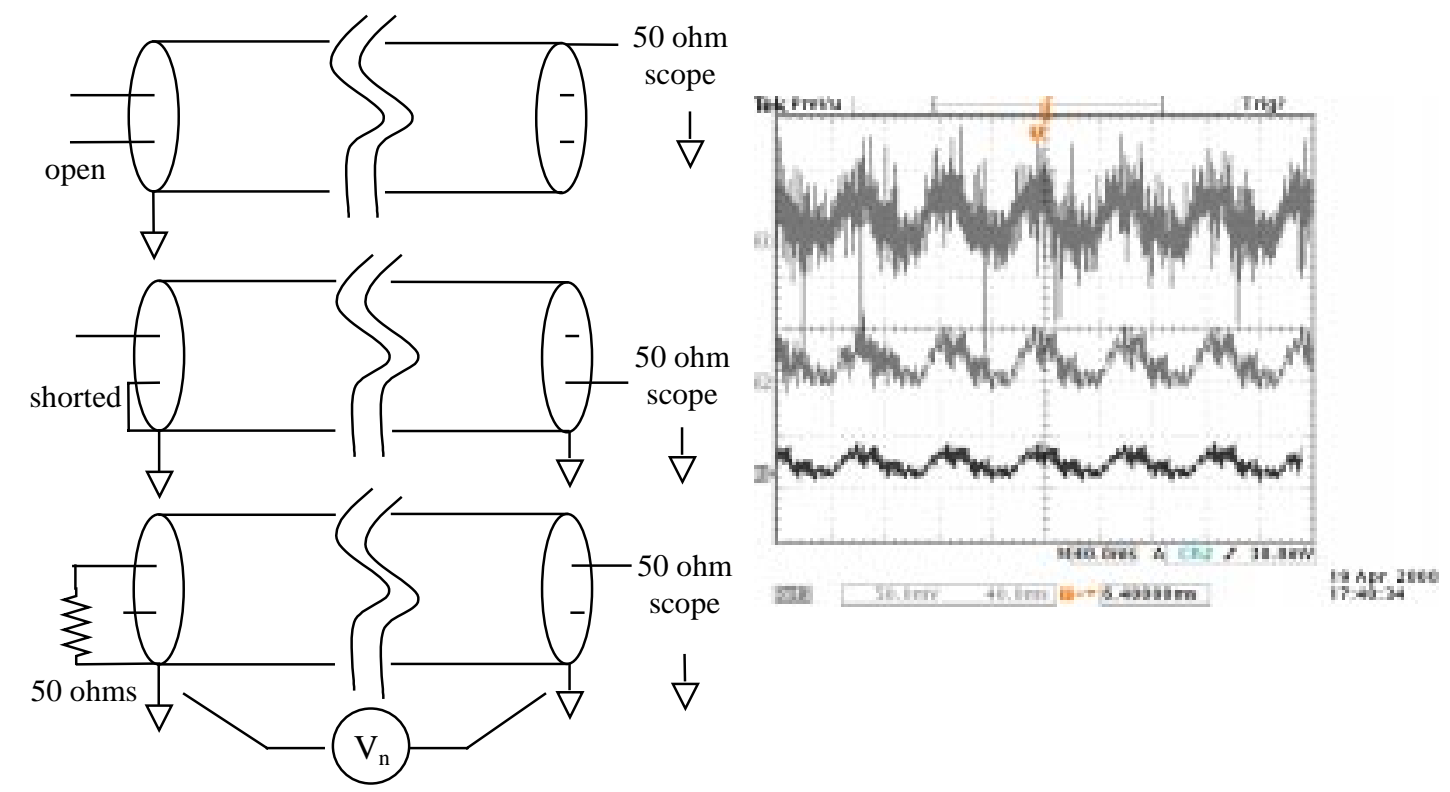

FIGURE 10. Noise on long twinax, various configurations with far end shield grounded. Top: noise on shield. Middle: either center into high $50 \mathrm{ohms}$ with far end tied to shield. Bottom: either center conductor into $50 \mathrm{ohms}$ with far end terminated to ground. (each $50 \mathrm{mV} / \mathrm{div}$ and $40 \mathrm{mS} / \mathrm{div}$ )

Fermilab Booster. The primary frequency component of the noise visible at the $40 \mathrm{msec} / \mathrm{div}$ scope speed is $15 \mathrm{~Hz}$, the frequency of the Booster main magnet system.

Figure 7 shows that low frequency noise even on a long coaxial cable can be small with a single point ground. Noise voltage between local and remote grounds couples into the load only through stray capacitance. Low frequencies are strongly suppressed 
by the capacitive reactance, but high frequencies pass readily. The short noise burst visible in Figure 7 is a $30-50 \mathrm{Mhz}$ signal due to beam circulating in the machine!

Figure 8 shows situations with the cable shield grounded at the remote end. The remote termination can be considered as a zero volt $50 \mathrm{ohm}$ impedance signal source between center and shield. The first trace displays the noise voltage between local and remote grounds viewed with the cable shield sampling the remote node. The second trace shows the noise voltage across the shield with both ends grounded and using the center conductor to sample the remote node. Notice that except for a difference in high frequency content, the noise voltage amplitude is essentially the same with and without the shield connected between the two grounds. This indicates that the noise source impedance is much smaller than the cable shield impedance, a fact that should not be surprising since the ground impedance is intended to be low and many conducting paths likely exist between the cable's two endpoints. The third trace shows that the observed noise signal is reduced to half amplitude when the receiving end is loaded in $50 \mathrm{ohms}$; the two resistances in the center conductor path simply form a voltage divider across the noise source.

Measurements of a long twinaxial cable with the shield grounded at both ends are shown in Figure 9. Capacitively coupled noise of significant amplitude is apparent on the center conductor when loaded with high impedance. The noise is largely absent when viewed into $50 \mathrm{ohms}$. This is another example of the voltage divider action, in this case between the rather large reactive coupling impedance and the scope impedance.
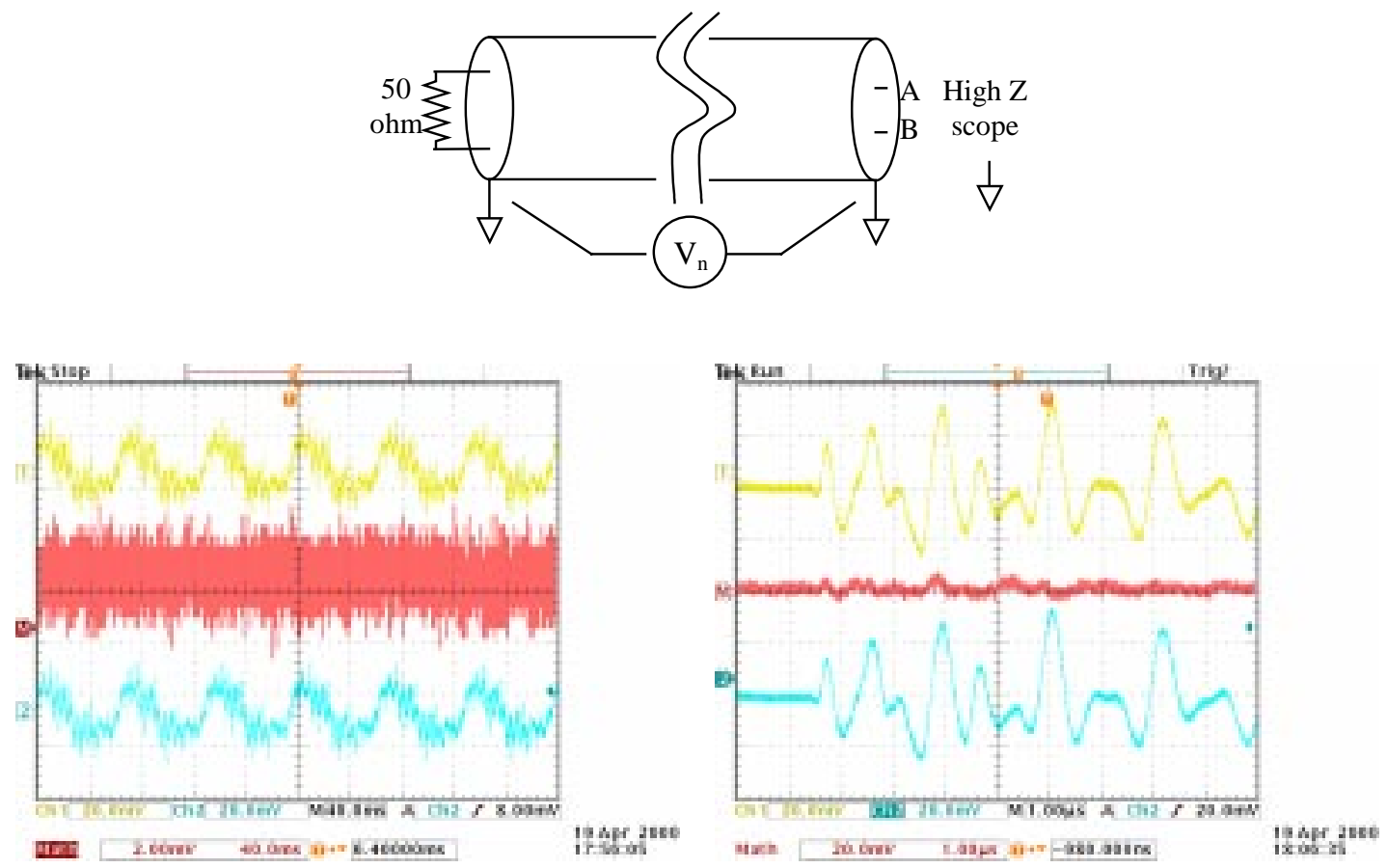

FIGURE 11. Differential noise on long twinax with far end shield grounded. Left: top and bottom, noise on either center conductor into $50 \mathrm{ohms}$ (each $20 \mathrm{mV} / \mathrm{div}$ ); center, difference $(2 \mathrm{mV} / \mathrm{div}$ ); (most of residual signal due to digital scope subtraction). Right: same signals faster time scale (each $20 \mathrm{mV} / \mathrm{div}$ and $1 \mathrm{uS} / \mathrm{div})$. 
With 50 ohms load, most of the voltage is dropped across the capacitance. The twinax cable measurements in Figure 10 compare with the similar coax measurements in Figure 8 . Note that the connection details differ slightly between the middle cases of the two figures, but the results are basically the same. This should be expected considering the voltage divider model and the very low source impedance of $\mathrm{V}_{\mathrm{N}}$.

The advantage of a floating, shielded signal source is manifested in Figure 11. Noise on either center conductor alone is the same as the first case of Figure 9, but the differential noise between the conductors is small. (Unfortunately, most of the noise apparent in the Fig. 11-Left difference trace was caused by the digital oscilloscope subtraction. Nearly the same noise appeared with both scope inputs grounded!) Even at higher frequencies, the differential noise is small (see Fig. 11-Right). The potential noise reduction advantage offered by the differential capability of twinaxial cable should be considered in applications where an ungrounded source is possible and the signal frequencies of interest are suitable $(\sim<100 \mathrm{Mhz})$ for transmission on such a cable. Note, however, that the advantage can be lost if the signal source is grounded or if the signal is not treated differentially at the receiver.

\section{Noise Reduction with a Magnetic Core}

It is common to find a coaxial cable wrapped several times on a high permeability toroidal magnetic core for the purpose of noise reduction. As shown in Figures 13 and 14 , the effectiveness of this practice, even at frequencies as low as $15 \mathrm{~Hz}$, can be significant. A casual explanation of the mechanism suggests that the inductive impedance added to the shield by the core reduces ground currents thereby diminishing the noise. But this explanation is curiously suspect! The noise voltage, already shown to manifest very low source impedance, is present between the two ends of the cable regardless of the shield current or impedance. Higher shield impedance cannot reduce that voltage. How is it that reduced shield current will improve the noise situation? Imagine that a cable shield with zero resistance could be installed. High ground current would flow, but the noise voltage would drop to zero and not appear in the signal load. If this lower shield impedance reduces the noise, how can it be that higher impedance would do the same?

The dilemma is solved by a different

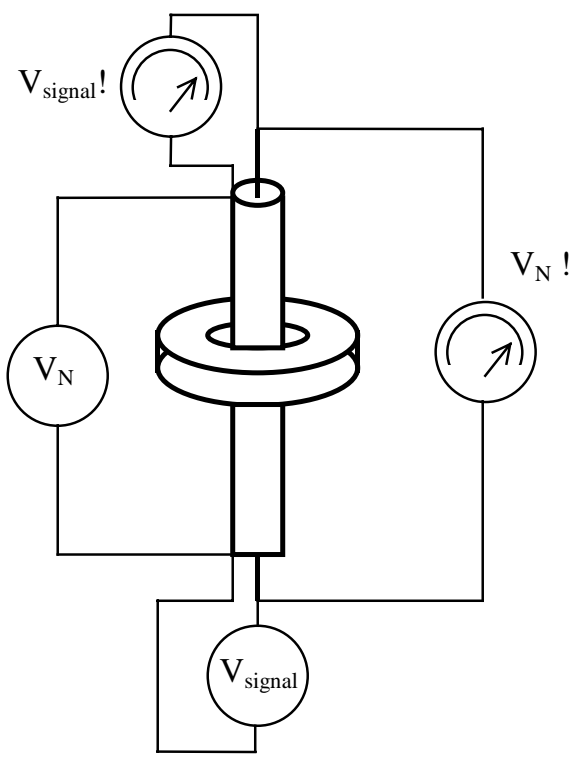
explanation, one that describes the core as forming a transformer between the coaxial shield and center conductor, coupling the noise voltage equally to both circuit legs. Consider Figure 12 showing a transformer core with two windings, one the shield of a coax cable and the other the center conductor of the same cable. Shield and center conductor, each looping the core N 

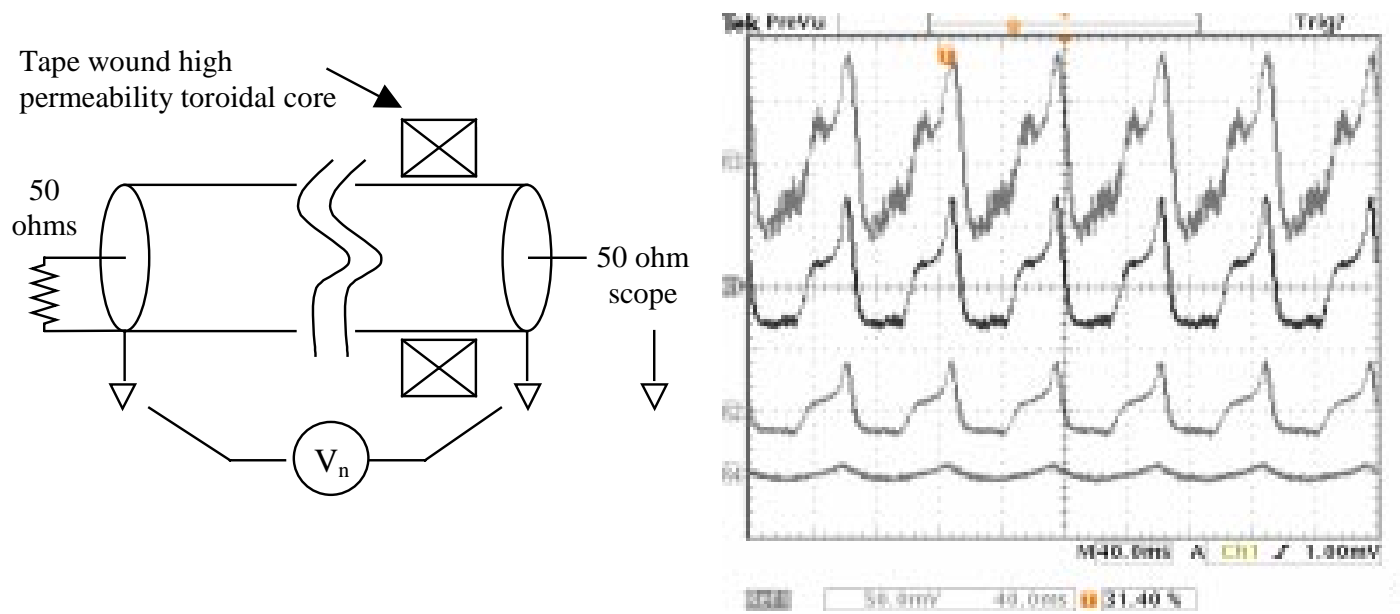

FIGURE 13. Effect of noise reduction core. Top: noise on long RG8 cable with no toroidal core. Second: 5 turns coax through toroid. Third: 10 turns. Fourth: many turns. (each $50 \mathrm{mV} / \mathrm{div}$ and $40 \mathrm{mS} / \mathrm{div})$
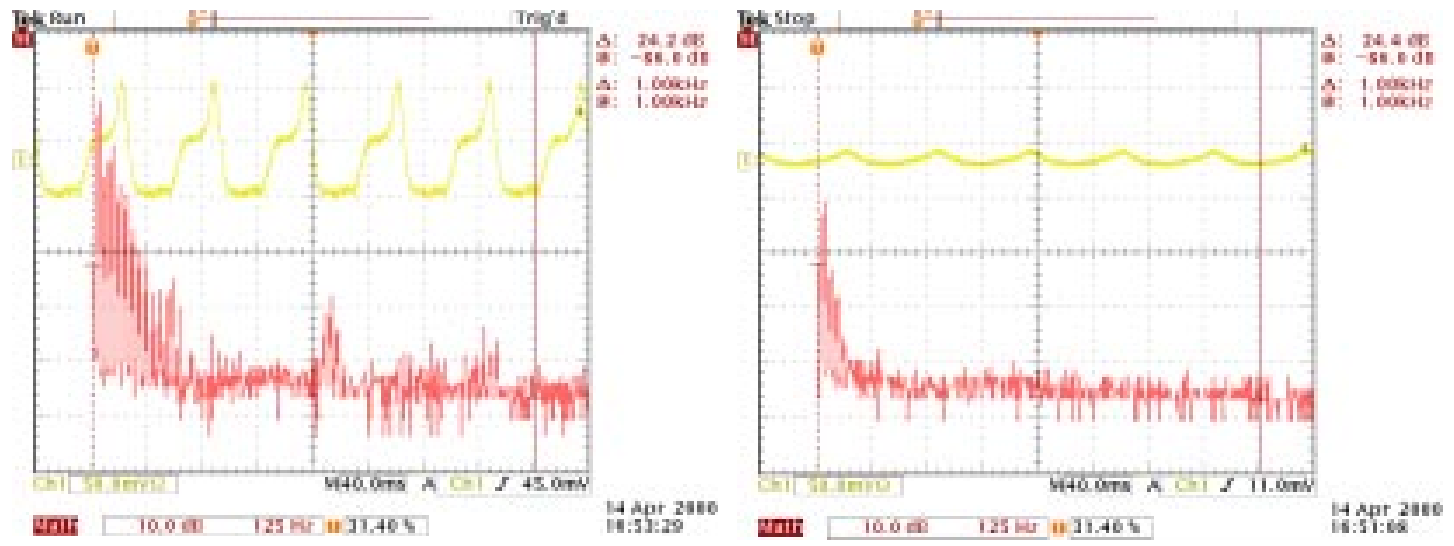

FIGURE 14. Noise voltage and spectrum for long RG8 cable with toroidal core. Left: 5 turns on core. Right: many turns on core. (each top trace $50 \mathrm{mV} / \mathrm{div}$ and $40 \mathrm{mS} / \mathrm{div}$ ) (each bottom trace $10 \mathrm{~dB} / \mathrm{div}$ and $125 \mathrm{~Hz} / \mathrm{div})$

times (one time depicted), link the same magnetic flux in core and therefore manifest the same induced voltage. At frequencies above $\omega=R / L$, where $R$ is the shield resistance and $\mathrm{L}$ is the inductance of the shield winding on the core, that voltage on the shield winding approaches a value equal to $\mathrm{V}_{\text {noise. }}$ The transformer action forces the same voltage to appear on the other winding, i.e. the center conductor, and the end-toend voltage on the center conductor will be identically equal to that of the shield, in this case $\mathrm{V}_{\text {noise }}$ ! Therefore the differential shield-to-center voltage at each end of the cable can be simultaneously independent of the noise voltage. The loop equation for a 
normal signal, $\mathrm{V}_{\mathrm{s}}$, through the coax includes $\mathrm{V}_{\text {noise }}$ once along the shield and then once again but with the opposite polarity along the center conductor. $\mathrm{V}_{\text {noise }}$ cancels along the normal coaxial signal conduction path and does not contaminate the desired signal! Quite satisfyingly, low shield resistance now appears as a good thing; lower resistance reduces the corner frequency at which the transformer action becomes effective. Dilemma resolved! Note that desired signals propagating inside coax, with shield and center conductor currents equal and opposite, are in no way be influenced by the core. With no net current presented to it, the core is effectively eliminated from the picture.

The effect of a noise reduction core with various numbers of turns for the same RG8 coaxial cable previously measured is shown in Figure 13. The corresponding noise spectra for the five-turn and the many turn cases are displayed in Figure 14. Note that the larger number of turns enhances noise attenuation at lower frequencies. This example demonstrates noise reduction performance achievable with a high permeability core on a coaxial cable at moderately low frequencies, less than about 110Mhz. Other materials such as suitable ferrites may be used at higher frequencies where they can provide higher quality inductive reactance than tape cores. In fact multiple cores of differing materials may be stacked on the same cable for improved results. However, for frequencies above a few tens of megahertz, the quality of the cable itself will have an even greater impact on shielding effectiveness.

\section{Local Noise}

To this point, only noise pick-up over long cables has been considered. Local noise sources can also be important. The top case in Figure 15 shows noise on the long RG8 coax due to the Booster extraction kicker power supply, a fast rise-time 1.6usec pulsed system, in the immediate vicinity of the receiving end of the cable. Even with many cable turns on the noise reduction core, this fast local noise is large. Compare top trace of Figure 15 at $20 \mathrm{mV} /$ div to bottom trace of Figure 13 at $50 \mathrm{mV} / \mathrm{div}$.

The second and third cases in Figure 15 demonstrate that this noise comes from a strong, local source. Trace two shows the noise picked up on just a twelve-inch length of RG58 cable grounded and terminated in $50 \mathrm{ohms}$ at both the scope and the opposite end. Trace three shows results for a twelve-inch length of 0.141 " semi-rigid copper coax line with SMA connectors similarly grounded and terminated. The superior noise rejection performance of the semi-rigid cable is another example of the skin effect that was described earlier. At high frequency, voltages and currents expressed on the outer surface of the coaxial outer conductor are attenuated before reaching the inner surface of that same conductor where they might couple into the normal signal path. The higher conductance and greater thickness of the semi-rigid shield relative to the RG58 shield yields more skin depths effective thickness and therefore better shielding. It is easily demonstrated in a situation like this that even loose connections seriously deteriorate noise performance of a cable system. Conclusion - High quality cable and solid connections are necessary to win the noise battle at high frequencies!

The data presented in Figure 15 was obtained with the Booster beam switched off. Figures 16 and 17 show similar measurements of the same coaxial cables, as well as that of a twelve-inch open wire, when beam is circulating. The revolution period of beam in the machine is $1.6 u s e c$ and all but about three of the eighty-four $53 \mathrm{Mhz}$ 


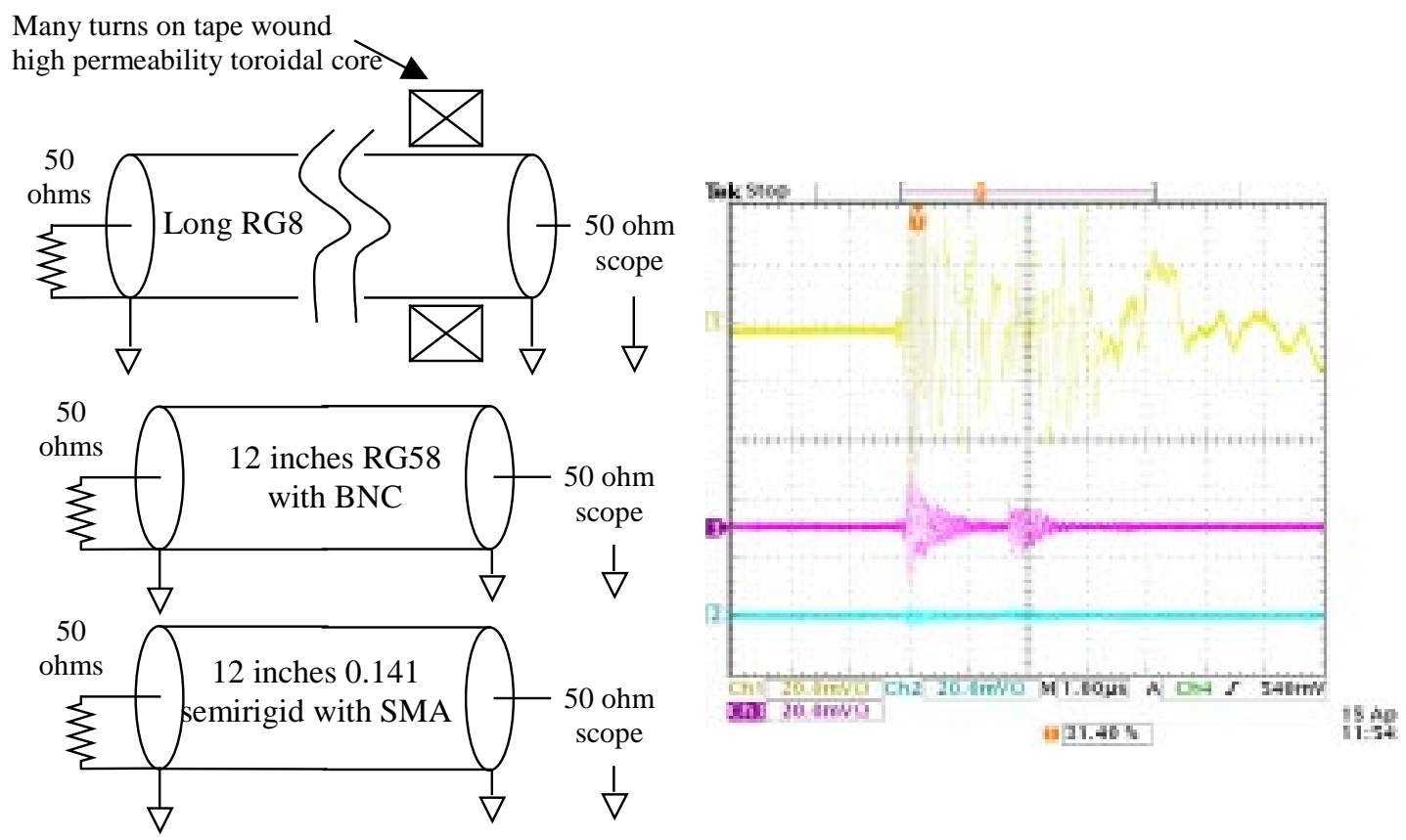

FIGURE 15. Local noise pick-up sensitivities of different cables. Top: same long RG8 with many turns on core. Middle: 12 inch length of RG58 with BNC connectors, terminated and grounded at each end. Bottom: 12 inch length of 0.141 semi-rigid coax with SMA connectors, terminated and grounded at each end. (each $20 \mathrm{mV} / \mathrm{div}$ and $1 \mathrm{uS} / \mathrm{div}$ )

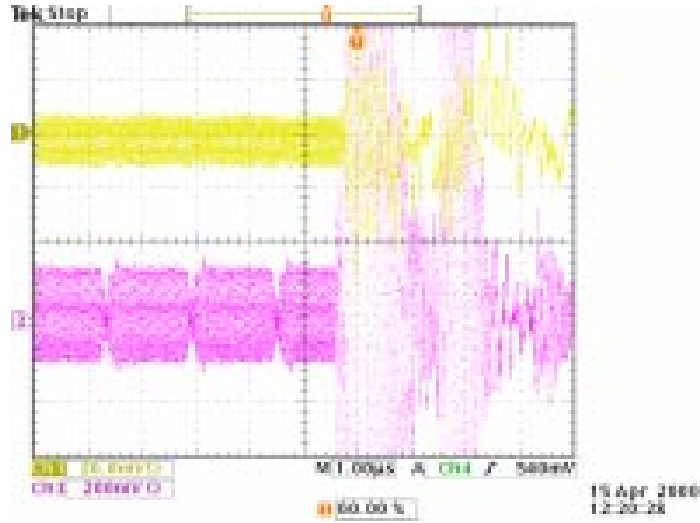

Figure 16. Noise pick-up with beam running; note 1.6 usec beam revolution frequency. Top: long RG8 as in Fig. 15 (20mV/div). Bottom: open 12" wire (200mV/div). (each 1usec/div)

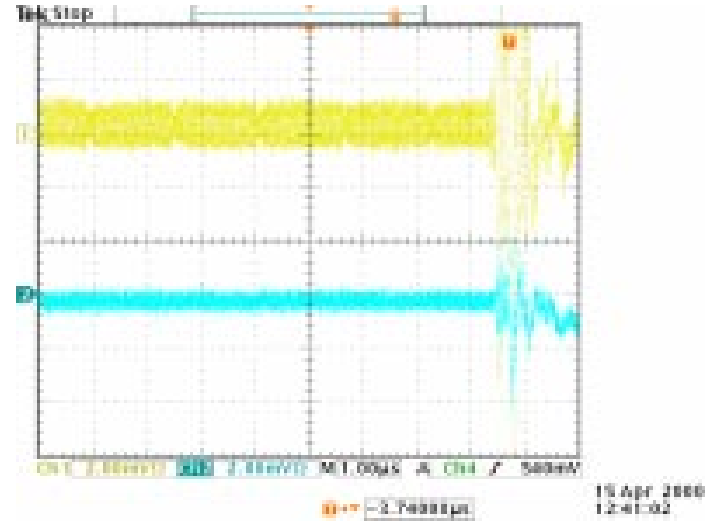

Figure 17. Noise pick-up with beam running; note 1.6 usec beam revolution frequency. Top: 12" RG58 as in Fig. 15 (2mV/div). Bottom: 12" 0.141 semi-rigid as in Fig. 15 (2mV/div). (each 1usec/div) 
buckets in the machine are filled. This structure is visible on scope traces in Figures 16 and 17. The beam rf signal picked up by the cables is radiated from unshielded gaps and ceramic kicker beam tubes in the machine in a manner as described earlier in the ceramic gap discussion. Even the beam makes noise, so it is proper to conscientiously shield all gaps!

\section{A SUCCESSFUL BEAM CURRENT MONITOR INSTALLATION}

As a working example, a beam current monitor installation used in the Fermilab $8 \mathrm{GeV}$ beam transport line is depicted in Figure 18. The system provides accurate measurement of the charge in each 1.6 usec beam pulse injected into the transport line. High fidelity representation of the macropulse shape is required, but resolution of the individual $53 \mathrm{Mhz}$ bunches is not. A system bandwidth of only a few megahertz is acceptable to meet signal observation requirements. Ultimately the signal is processed by an analog integrate and hold circuit before presentation to the control system.

The current transformer itself is a commercially available model featuring an isolated output winding and a metallic case for shielding. Twinax cable is utilized to transport the signal about 50 meters to the processing electronics. (Noise reduction core is optional.) The twin signal conductors, totally contained within a Faraday enclosure formed by the transformer case, cable shield, and metal electronics chassis, are grounded only at the differential receiving amplifier input. The amplifier circuit (see Fig. 19), based on the Analog Devices AD830, is designed with suitable bandwidth to meet signal processing requirements and good common mode rejection to further attenuate noise. Note that the amplifier is not DC coupled to the transformer. If this seems inappropriate, remember that the transformer does not DC couple to the beam in any case, so nothing is lost! The measured frequency response and common mode rejection ratio of the amplifier circuit are given in Figures 20 and 21.

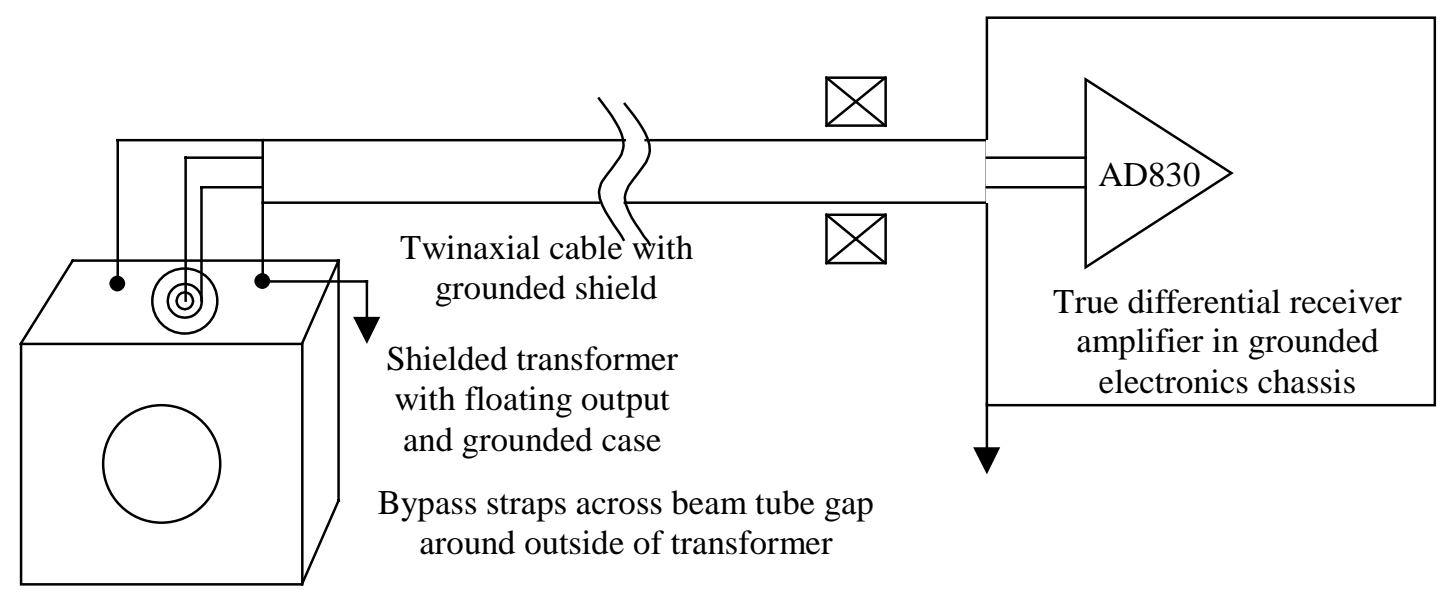

FIGURE 18. A successful beam current monitor installation example. 


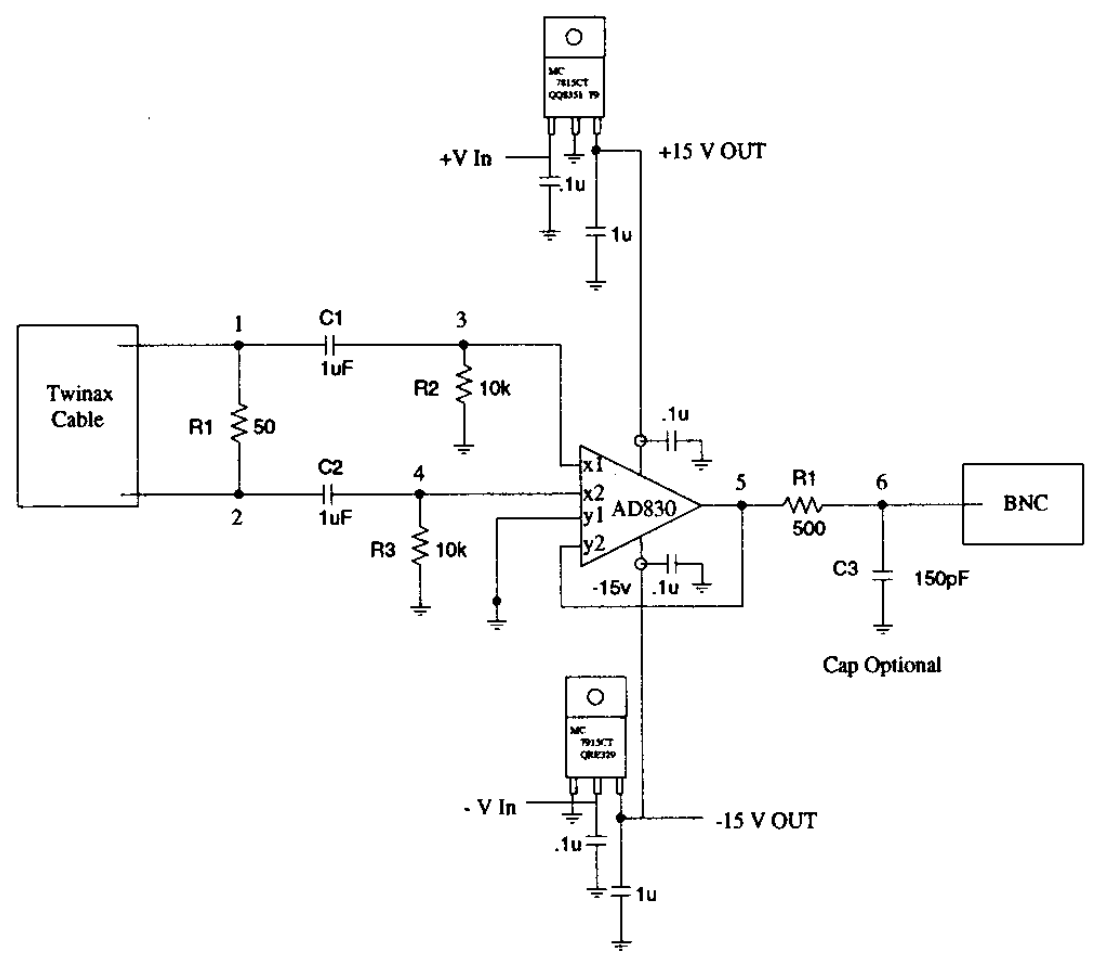

FIGURE 19. AD830 differential amplifier circuit used on Fermilab $8 \mathrm{GeV}$ beamline current monitor.

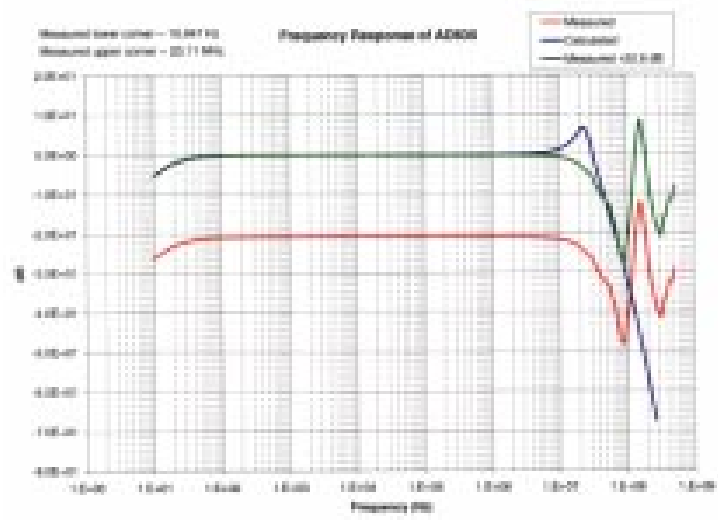

FIGURE 20. AD830 based amplifier frequency response.

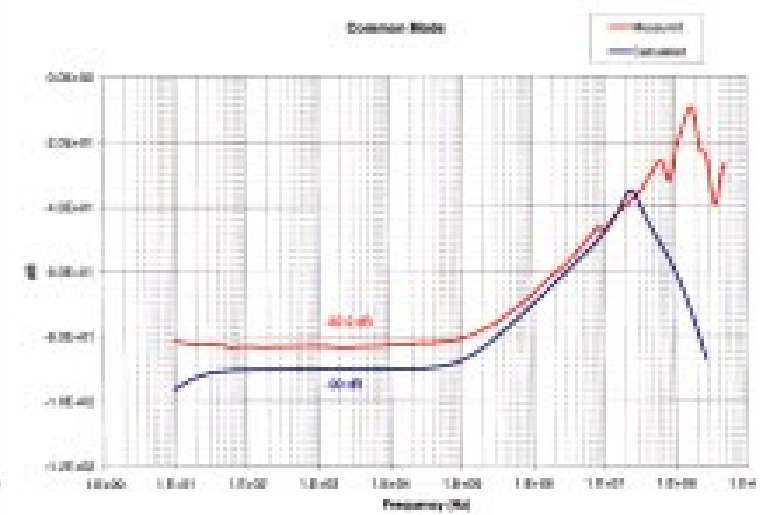

FIGURE 21. AD830 based amplifier common mode rejection performance.

This installation performs well for its required purpose and serves here only as one example of a practical solution to a specific set of requirements. Clearly, different requirements of other applications will logically and necessarily lead to alternative designs. For example, a system expected to provide a bandwidth approaching 
$100 \mathrm{Mhz}$ would probably require a different transformer, high quality coaxial cable in place of twinax, and a different amplifier circuit to maintain signal integrity.

\section{MISCELLANEOUS COMMENTS}

Signal processing requirements will obviously depend on the specific beam current monitor application. For a relatively flat-topped current pulse or a fixed signal shape (e.g. for long bunch train down transport line or for ICT output), a single sample of the signal amplitude at a known time may be sufficient. To record time shape of a current pulse, fast digitization may be required. Signal integration is required if measurement of the total charge of variable shape or duration beam pulses is the objective. Often, amplification is necessary as the first stage of signal processing. The goal of any processing circuit is to acquire and preserve the desired beam information with credible and reliable accuracy.

In the processing circuit design, it is important to keep in mind that the signal from a beam current transformer (DCCT excluded) is not DC coupled. Timing issues will be important in establishing the accuracy and reliability of any static processed output. Active baseline restoration may be used to establish a DC reference at a single point in time, but baseline slope may remain a problem. In general, the transformer signal baseline level and slope is dependent on previous beam pulses for times up to several transformer time constants. Best integration results are achieved when the transformer time constant is long relative to the duration of the beam pulse to be measured and with the integration window as narrow as possible. Remember, the integral of a nonperiodic AC signal over an indefinite length of time is identically zero.

The source impedance presented by the transformer must be considered; it will not be the same high impedance as the beam itself. Many transformers designed for high frequency operation include an internal $50 \mathrm{ohm}$ load and thus present a $50 \mathrm{ohm}$ source impedance over their useful range. Any other current transformer will present reactive source impedance equal to the winding inductance. System calibration is directly affected by cable attenuation and by the terminating impedance at the receiving end of the cable.

A single turn calibration winding is often included in a beam current monitoring system. The calibration winding will have minimal incidental effect on the system transfer function provided it is not shorted. A transformer secondary winding presents itself to the primary as an impedance proportional to the secondary load impedance and inversely proportional to the square of the secondary turns. A typical 1 volt/amp transformer with 50 turns and an internal 50 load reflects back to the beam as 0.02 ohms. A single turn calibration winding on the same transformer, even terminated in $50 \mathrm{ohms}$, reflects back as 50 ohms. The loading due to the calibration winding is only a 1:2500 effect. If using a calibration winding to calibrate for fast pulses, these effects must be considered: a) the value of any resistive termination of the calibration cable, b) leakage inductance of the calibration winding through transformer, and c) any capacitance of the calibration winding to the transformer and case. Where possible it may be useful to provide a return cable to bring the calibration current back to the source to facilitate measurement of the current actually passing through monitor. 
Proper shielding and noise considerations must always be afforded to calibration cabling. Any noise coupling into the calibration winding will be indistinguishable from real signal by the current monitor!

\section{ACKNOWLEDGMENTS}

The author acknowledges thought provoking and enlightening conversations with Jim Crisp and Peter Prieto, both of Fermilab. Jim and his summer student, Mike Reid, provided the gap impedance measurement data and the AD830 differential amplifier circuit design and performance figures.

\section{SELECTED BIW AND DIPAC PAPERS ON BEAM CURRENT MONITORING AND CLOSELY RELATED TOPICS}

First Workshop, 1989, BNL, Upton, NY --- Accelerator Instrumentation, edited by E. R. Beadle and V. J. Castillo, AIP Conference Proceedings 212, New York: American Institute of Physics, 1990.

Talman, R., "Beam Current Monitors", pp. 1-25.

Webber, R. C., "Longitudinal Emittance: An Introduction to the Concept and Survey of Measurement Techniques Including Design of a Wall Current Monitor", pp. 85-125.

Second Workshop, 1990, Fermilab, Batavia, IL --- Accelerator Instrumentation, Second Annual Workshop, edited by E. S. McCrory, AIP Conference Proceedings 229, New York: American Institute of Physics, 1991.

No papers specific to topic.

Third Workshop, 1991, CEBAF, Newport News, VA --- Accelerator Instrumentation, Third Annual Workshop, edited by W. Barry and P. Kloeppel, AIP Conference Proceedings 252, New York: American Institute of Physics, 1992.

Gallegos, F. R., "Development of a Low Intensity Current Monitor System”, pp. 1-20.

Kerns, Q. A., "Grounding and Shielding in the Accelerator Environment", pp. 43-64.

Unser, K. B., "The Parametric Current Transformer, a Beam Current Monitor Developed for LEP", pp. 266-275.

Fourth Workshop, 1992, LBL, Berkeley, CA --- Accelerator Instrumentation, Fourth Annual Workshop, edited by J. A. Hinkson and G. Stover, AIP Conference Proceedings 281, New York: American Institute of Physics, 1993.

Berners, D., and Reginato, L. L., "Beam Position and Total Current Monitor for Heavy Ion Fusion Beams", pp. 168-174.

Schutte, W., and Unser, K. B., "Beam Current and Beam Lifetime Measurements at the HERA Proton Storage Ring", pp. 225-233.

Wang, X., "Design and Initial Tests of Beam Current Monitoring Systems for the APS Transport Lines", pp. 234-241.

Fifth Workshop, 1993, Sante Fe, NM --- Beam Instrumentation Workshop, edited by R. E. Shafer, AIP Conference Proceedings 319, New York: American Institute of Physics, 1994.

Yin, Y., et al., "An In-Vacuum Wall Current Monitor and Low Cost Signal Sampling System", pp. 363-371. 
Sixth Workshop, 1994, Vancouver, BC, Canada --- Beam Instrumentation Workshop, edited by G. H. MacKenzie et al., AIP Conference Proceedings 333, New York: American Institute of Physics, 1995.

Webber, R. C., "Charged Particle Beam Current Monitoring Tutorial”, pp. 3-23.

Wang, X., "Ultrafast, High Precision Gated Integrator", pp. 260-266.

Wang, X., et al., "Design and Commissioning of the APS Beam Charge and Current Monitors", pp. 267-273.

Ball, M., and Hamilton, B., "Current Monitors for Intensity Modulated Beams”, pp. 281-286.

Heeg, P., et al., "Intensity Measurements of Slowly Extracted Heavy Ion Beams from the SIS", pp. 287-293.

Peterson, D. W., "An Overview of High Input Impedance Buffer Amplifiers for Wide Bandwidth Signals", pp. 356-362.

Seventh Workshop, 1996, ANL-APS, Argonne, IL --- Beam Instrumentation, Proceedings of the Seventh Workshop, edited by A. H. Lumpkin and C. E. Eyberger, AIP Conference Proceedings 390, New York: American Institute of Physics, 1997.

Seimann, R. H., "Spectral Analysis of Relativistic Bunched Beams", pp. 3-22.

Corlett, J. N., "Impedance of Accelerator Components", pp. 66-88.

Fessenden, T. J., "Diagnostics for Inductin Accelerators", pp. 110-129.

Vogel, G., et al., "A Multi-Batch Fast Bunch Integrator for the Fermilab Main Ring”, pp. 414-421.

Forck, P., et al., "Intensity Measurement of High-Energy Heavy Ions at the GSI Facility", pp. 422429.

Abildskov, F., and Moller, S. P., "Bunched-Beam Measurements of Very Small Currents at ASTRID”, pp. 536-543.

Eighth Workshop, 1998, SLAC, Stanford, CA --- Beam Instrumentation Workshop, edited by R. O. Hettel et al., AIP Conference Proceedings 451, New York: American Institute of Physics, 1998.

Peters, A., et al., "A Cryogenic Current Comparator for the Absolute Measurement of nA Beams", pp. 163-182.

Fellenz, B., and Crisp, J., “An Improved Resistive Wall Monitor”, pp. 446-453.

Schneider, N., "Beam Current Monitors at the UNILAC", pp. 502-506.

DIPAC, 1993, Montreux, Switzerland --- Proceedings of the First European Workshop on Beam Diagnostics and Instrumentation for Particle Accelerators, CERN PS/93-35 (BD), CERN SL/93-35 (BI), 1993.

Trier, J. O., and Derikum, K., "High-Accuracy Absolute Charge Measurement of a Low-intensity Electron Beam", pp. 91-95.

Peters, A., et al., "A Cryogenic Current Comparator for Low Intensity Beams”, pp. 100-104.

Unser, K. B., "Recent Advances in Beam Current Transformer Technology and Avenues for further Developments", pp. 105-109.

DIPAC, 1995, Travemunde, Germany --- 2nd European Workshop on Beam Diagnostics and Instrumentation for Particle Accelerators, DESY M-95 07, 1993.

Peters, A., et al., "A New d.c. SQUID Current Comparator for Low Intensity Ion Beams", pp. 136138.

Cherepanov, V. P., "Image Current Monitor for Bunched Beam Parameters Measurements", pp. 142-144. 